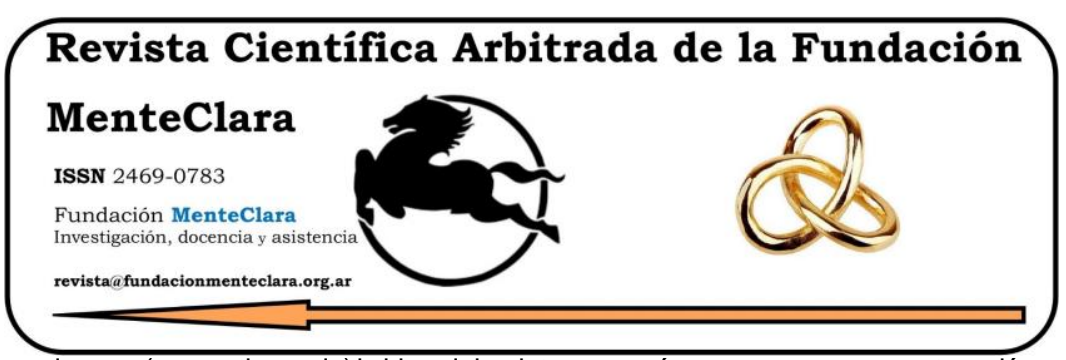

Artículos atravesados por (o cuestionando) la idea del sujeto -y su género- como una construcción psicobiológica de la cultura. Articles driven by (or questioning) the idea of the subject -and their gender- as a cultural psychobiological construction.

Vol. 7 (2022), enero-diciembre ISSN 2469-0783

https: / / datahub.io/dataset/2022-7-e259

\title{
OPTIMISMO Y RESILIENCIA EN ADOLESCENTES
}

\section{OPTIMISM AND RESILIENCE IN ADOLESCENTS}

Maria del Rosario Richards maria.richards@uflo.edu.ar

Universidad de Flores, Psicología, Psicología de la Salud, Argentina.

Cómo citar este artículo / Citation: Richards, M. (2022). Optimismo y Resiliencia en Adolescentes. Revista Científica Arbitrada de la Fundación MenteClara, Vol. 7 (259). DOI: https://doi.org/ 10.32351/rca.v7.259

Copyright: (C) 2022 RCAFMC. Este artículo de acce so abierto es distribuido bajo los términos de la licencia Creative Commons Attribution 4.0 International License (CC BY 4.0). Recibido: 18/01/2022. Aceptado: 04/02/2022 Publicación online: 11/02/2022

Conflicto de intereses: Ninguno que declarar.

\section{Resumen}

La psicología positiva propone un cambio del enfoque centrado en la patologia, en la enfermedad y en las emociones negativas, a un cambio centrado en las fortalezas, las virtudes y emociones positivas, así como en numerosos constructos relacionados con el bienestar de los individuos. El objetivo de este trabajo es desarrollar el constructo optimismo y como se relaciona a la capacidad de los adolescentes para la superación de situaciones adversas, es decir, la resiliencia. Si bien la búsqueda ha sido dificultosa el interés en estos temas desde la Psicología Positiva y su impacto en la salud mental es cada vez mayor. A partir de los artículos estudiados, se demuestra que el optimismo tiene influencia significativa en la resiliencia de los adolescentes. Aquellos adolescentes que se presentan como optimistas, van a tener personalidad resiliente lo cual podría ser un factor que los protege de las adversidades que se presentan en esta etapa tan especial de su desarrollo. El optimismo a su vez, se puede desarrollar como cualquier otra fortaleza humana, lo cual también se vería reflejado a futuro en la propia resiliencia. 


\begin{abstract}
Positive psychology proposes a change from an approach focused on pathology, illness, and negative emotions, to strengths, virtues, and positive emotions, as well as numerous constructs related to the well-being of individuals. The objective of this work is to develop the optimism construct and how it is related to adolescents' ability to overcome adverse situations, that is, resilience. Although the search has been difficult, the interest in these topics from Positive Psychology and its impact on mental health is increasing. From the articles studied, it is shown that optimism has a significant influence on the resilience of adolescents. Those adolescents who present themselves as optimistic will have a resilient personality, which could be a factor that protects them from the adversities that can occur at this very special stage of their development. Optimism, in turn, can be developed like any other human strength, which would also be reflected in the future in one's own resilience.
\end{abstract}

Palabras Claves: resiliencia; optimismo; adolescentes

Keyw ords: resilience; optimism; adole scents 


\section{Introducción}

Seligman (2017) nos habla de una epidemia juvenil en torno a la depresión de los adolescentes y como ha crecido la depresión en general de forma drástica en los últimos 40 años en todos los países ricos del mundo. Con el fin de entender un poco más la personalidad de los adolescentes, vamos a tratar en este trabajo la relación existente, si es que hay, entre el optimismo y la resiliencia en este grupo. Y descubrir si alguno influye sobre el otro significativamente.

Si hacemos un breve recorrido de la historia de la Psicología de la salud vemos que ya desde el año 1948 la Organización Mundial de la Salud definió como concepto de salud al estado de completo bienestar físico, mental y social y no meramente la ausencia de la enfermedad (OMS, 1948). Desde este momento se empezó a cambiar la mirada desde la enfermedad hacia la prevención de la salud, enfocándose más en los aspectos positivos de la salud y no tanto en la enfermedad. De la mano de la Psicologia Positiva, se fue dando lugar a constructos que fomentan el desarrollo del bienestar psicológico y por lo tanto aumentar la felicidad de las personas. Seligman en su libro "La Autentica Felicidad" (2017) nos presenta 24 fortalezas que se pueden desarrollar para aumentar nuestro bienestar. Entre ellas se encuentra el optimismo que es nuestra primera variable de estudio. Se desarrollará la definición de optimismo disposicional, como influye el optimismo en la vida de las personas y algunos estudios al respecto.

Por otro lado, se definió entender y desarrollar el constructo de resiliencia como otra variable de la personalidad. Así, también entender que es la resiliencia y como son las personas resilientes. Cada vez se encuentran más estudios sobre la resiliencia, en especial en los últimos años (Sagone \& Caroli, 2015) (Finez-SIlva, Moran-Astorga, \& Urchaga- 
Litago, 2019) (Gomez Molinero, Zayas, Ruiz Gonzalez, \& Guil, 2018) (Guzman \& Cardenas, 2021).

\section{Optimismo}

El optimismo según Abramson, Seligmany Teasdale (1978) se entiende como una forma de explicar las respuestas de afrontamiento a los eventos negativos que les suceden a las personas en sus vidas. De esta manera definieron dos estilos explicativos, el optimista y el pesimista. Siendo el Estilo Explicativo Pesimista (EEP) como la tendencia a explicar los malos sucesos o eventos negativos ocurridos en la vida cotidiana con una causa interna a uno mismo, estable en el tiempo y con un efecto global en todos los ámbitos de la vida de la persona. Por el otro lado, el Estilo Explicativo Optimista (EEO) se refiere a la tendencia explicar los malos sucesos con una causa externa a uno mismo, inestable en el tiempo y especifico de ese ámbito concreto que le afecte (Peterson \& Seligman, 1984). Por lo tanto, como dice Seligman (1998), las personas optimistas tienden a interpretar que sus problemas son pasajeros, controlables y propios de una situación. Las personas pesimistas, por otro lado, creen que los problemas van a durar mucho tiempo, no podrán controlarlos y socavaran todo lo que hagan (globalidad).

Por otro lado, tenemos el optimismo disposicional definido por Scheier y Carver (1987) como la expectativa o creencia estable, generalizada de que en la vida ocurrirán cosas positivas. Si las expectativas de las personas son favorables, entonces aumentan los esfuerzos para alcanzar objetivos, de lo contrario, si las expectativas son desfavorables disminuyen dichos esfuerzos a tal punto de desentenderse por completo de los objetivos. 
Los estudios realizados a los largo del tiempo sobre el optimismo tratan de buscar la razón por la cual una persona es más optimista que otra y cuáles son las variables que influyen. Según un estudio de Lavarello (2017) no hay diferencia significativa en los niveles de optimismo entre adultos en algunos países latinoamericanos, aunque si en niveles de pesimismo por ejemplo. Pero, como bien explican en su libro Psicología Positiva Aplicada, Vázquez y Hervas (2008) se puede decir que el nivel de bienestar de una persona y así también el nivel de optimismo y otras emociones positivas van a depender en un $50 \%$ por su biología (set-point), un $40 \%$ en lo que hace la persona y un $10 \%$ en las circunstancias que lo rodean. Por esto se puede ver que la vida inflige los mismos contratiempos y tragedias en el optimista como en el pesimista (circunstancia), pero el optimista las resiste mejor ya sea por su biología o por la actitud que desarrolle (Abramson, Seligman, \& Teasdale, 1978).

Ahora bien, pareciera que ser optimista mejora la salud en general y trae menos vulnerabilidad a las enfermedades fisicas (Kamen \& Seligman, 1987) además de proveer mejor funcionamiento del sistema inmunológico (Peterson \& De Avila, 1995). Podemos de esta manera afirmar que el optimismo influye positivamente en la vida de las personas, incluso les permite mantener el bienestar psicológico antes adversidades (Hervas \& Vazquez, 2008).

\section{Resiliencia}

Desde la psicología, la resiliencia se entiende como la capacidad que tiene el ser humano de superar la adversidad y salir fortalecido de ella. El término resiliencia proviene del latín resilio, que indica la capacidad de saltar hacia atrás o rebotar, refiriéndose a la capacidad de los materiales para recibir impactos y recuperarse, volviendo así al estado 
anterior a la deformación. Por lo tanto, podemos decir que las personas resilientes son aquellas que consiguen mantenerse en equilibrio sin que afecte a su rendimientoy a su vida cotidiana (Finez-SIlva, Moran-Astorga, \& Urchaga-Litago, 2019). Además, son capaces de aprender y crecer a partir de esas experiencias adversas (Bonanno, et al., 2002).

Durante los últimos años, se llevaron a cabo estudios para conocer un poco más el constructo resiliencia, tanto en adultos como adolescentes $\mathrm{y}$ en diferentes contextos sociales intentando encontrar similitudes y diferencias en aquellas personas que son o no resilientes (Finez-SIlva, Moran-Astorga, \& Urchaga-Litago, 2019), (Irurzun, Mezzadra, \& Preuss, 2017), (Guzman \& Cardenas, 2021), (Fuentes \& Medina, 2012).

Uno de ellos es el de Fínez et al., (2019) donde el objetivo era de verificar si la edad y el sexo impactaban significativamente en la característica resiliencia. Dentro de los resultados de este estudio, vieron que en función de la edad los adultos son más resilientes que los adolescentes. La resiliencia va aumentando desde la adolescencia hasta llegar a la etapa de adultos-jóvenes y sigue en aumento hasta la etapa de adultez. En cambio, no encontraron diferencias significativas en función del sexo, pero pudiendo concluir que la variabilidad en resiliencia es considerablemente diferente entre los adolescentes: los varones son más resilientes que las mujeres en este grupo. Por el contrario, en el grupo de adultos aparecen las mujeres como más resilientes que los varones.

Otros estudios como el de Irurzun et al., (2017) estudiaron la resiliencia desde una perspectiva psico-social, en donde en lugar de considerar que la resiliencia se define por las conductas individuales de superación, las cuales parecían ser casos puntuales y anecdóticos, sino que es un proceso más amplio en el cual intervienen patrones y características generales del individuo, así como puede ser la espiritualidad. 
En un artículo de Vinaccia et al., (2007) los autores señalan que los adolescentes resilientes presentan una mayor inteligencia y habilidad de resolución de problemas que los no resilientes. Según los autores Fergusson \& Lynskey (1996), esto significa que una condición necesaria, aunque no suficiente, para la resiliencia es poseer una capacidad intelectual igual o superior al promedio. También vieron que aquellos adolescentes que en la actualidad presentaban características resilientes habían sido catalogados como niños fáciles y de buen temperamento durante su infancia. Por lo tanto, podemos decir que un adolescente llega a ser resiliente no solo por su biología, sino también por el contexto de su niñez.

\section{Optimismo y su impacto en la resiliencia en adolescentes}

La adolescencia es la etapa entre la niñez y adultez donde el joven se enfrenta a retos y obstáculos significativos para él, enfrentándose al desarrollo, en primera instancia, de su identidad y a la necesidad de conseguir la independencia de la familia manteniendo al mismo tiempo la conexión y la pertenencia al grupo (Vinaccia, Quinceno, \& San Pedro, 2007). Siendo esta etapa, un momento de grandes cambios en la personalidad buscamos analizar si existe alguna relación entre el optimismo y la resiliencia en adolescentes

En su artículo de Emociones Positivas, María Luisa Vecina Jiménez (2006) explica citando a Bárbara Fredrickson (1998) que experimentar emociones positivas como puede ser la alegría del optimista es siempre agradable y placentero a corto plazo y tendría otros efectos beneficiosos más duraderos, en la medida en la que ello prepara a los individuos para tiempos futuros más duros, en definitiva, haciéndolos más resilientes. Nos explica que las emociones positivas ayudan a construir la resiliencia 
psicológica haciendo a las personas más resistentes frente a la adversidad.

En un estudio de Gómez et al. (2018) sobre alumnos universitarios, los resultados indicaban que el optimismo predecía significativamente niveles de resiliencia entre los alumnos. Esto significa que aquellos que tenian buenas expectativas sobre su futuro, enfrentaban la adversidad de manera más adaptativa. Los autores concluyen que el optimismo refleja actitudes positivas frente a circunstancias adversas estableciendo que el optimismo es uno de los aspectos más importantes para el desarrollo de la resiliencia.

Otro estudio en adolescentes de una universidad pública de Fuentes y Medina (2012), evidencia que los adolescentes resilientes son más optimistas que los no resilientes. Los sujetos optimistas manifestaron los hechos desagradables que les suceden como fruto de causas específicas y no algo generalizado como decía Seligman (2017). Respecto al sexo, no se encontraron diferencias en las variables resiliencia y optimismo. Los autores indagaron un poco más en la relación entre resiliencia y optimismo, y realizaron un análisis de correlación que mostró una relación directa entre ambas variables, concluyendo que una persona que es resiliente es capaz de enfrentarse a la adversidad de forma positiva, haciendo uso de diferentes recursos para superar el dolor y salir fortalecido de dichas circunstancias, es una persona más optimista.

\section{Conclusiones}

Como vimos en la introducción, Seligman nos hablaba de la epidemia sobre la depresión en jóvenes, pero nos da también herramientas para aumentar el bienestar a través del desarrollo de las fortalezas (2017). La investigación en resiliencia puede resultar muy útil para estos tiempos 
especialmente para conocer cuáles son los recursos psicológicos que poseen para salir adelante los adolescentes de nuestro contexto, como puede ser el desarrollo del optimismo.

Por otro lado, podemos ver que la investigación en niños, nos pueden predecir la resiliencia en la adolescencia y de esta manera poder influir en los aprendizajes iniciales que son fundamentales para la vida. Un niño optimista, probablemente se desarrolle en un adolescente resiliente.

En conclusión, estos estudios nos permiten afirmar que los adolescentes resilientes poseen optimismo, lo cual podría ser un factor que los protege de la depresión, al mismo tiempo de que potencia los recursos psicológicos de los individuos. El optimismo es un factor protector frente a la adversidad, a mayor optimismo, mayor resiliencia van a presentar los adolescentes. Y este optimismo a su vez, se puede desarrollar como cualquier otra fortaleza humana. Merece destacar la necesidad de seguir investigando igualmente la interdependencia de estas variables. 


\section{Referencias}

Abramson, L., Seligman, M. E., \& Teasdale, J. D. (1978). Learned helplessness in humans: critique and reformulation. Journal of abnormal psychology, 87.

Bonanno, G. A., Wortman, C. B., Lehman, D. R., Tweed, R. G., Haring, M., \& Sonnega, J. (2002). Resilience to loss and chronic grief: a prospective study from preloss to 18 -months postloss. Journal of personality and social psychology, 1150.

Diener, E., Lucas, R. E., \& Scollon, C. N. (2009). Beyond the hedonic treadmill: Revising the adaptation theory of well-being. The Science of well-being, 103-109.

Fergusson, D. M., \& Lynskey, M. T. (1996). Adole scent resilience to family adversity. Journal of Child Psychology and Phsychiatry, 281-292.

Finez-SIlva, M. J., Moran-Astorga, C., \& Urchaga-Litago, J. D. (2019). Resiliencia psicologica a traves de la edad y el sexo. International Journal of Developmental and Educational Phychology., 85-94.

Fredrickson, B. L. (1998). What good are positive emotions? Review of general psychology, 300-319.

Fuentes, N. I., \& Medina, J. L. (2012). Optimismo-pesimismoy resiliencia en adolescentes de una universidad pública. Revista Cientifica Multidisciplinaria de Prospectiva., 207-214.

Gancedo, M. (2008a). Historia de la Psicologia Positiva. Antecedentes, aportes y proyecciones. Buenos Aires: Lugar Editorial.

Gomez Molinero, R., Zayas, A., Ruiz Gonzalez, P., \& Guil, R. (2018). Optimism and resilience among university students. INFAD.

Gomez, C. M., \& Gundin, O. A. (2018). Resiliencia, optimismoy burnout academico en estudiantes universitarios. European Journal of Education and Psychology., 47-59.

Guzman, A. M., \& Cardenas, O. M. (2021). Resiliencia y cultura terapeutica en tiempos neoliberales: una exploracion de discursos de autoayuda. Quaderns de Psicologia., 2.

Hervas, G., \& Vazquez, C. (2008). Psicologia positiva aplicada. Bilbao: Biblioteca de Psicologia Desclee de Brouwer.

Irurzun, J. I., Mezzadra, J., \& Preuss, M. (2017). Resiliencia y Espiritualidad. Aportes para su estudio desde una perspectiva psicologica. Biblioteca Repositorio. Escuela de Tantra en España., 205-216.

Kamen, L. P., \& Seligman, M. E. (1987). Explanatory style and health. Current Psycology., 207-218.

Lavarello, J. R., Guerrero, E. E., \& Vergara, J. F. (2017). Niveles de Optimismo: Diferencias entre adultos de paises latinoamericanos. Universitas Pshycologica, 1-7.

OMS, O. (1948). Constitución OMS. Glosario de Promoción de la Salud.

Peterson, C., \& De Avila, M. E. (1995). Optimistic explanatory style and the perception of health problems. Journal of clinical Psychology., 128-132.

Peterson, C., \& Seligman, M. E. (1984). Causal explanations as a risk factor for depression: theory and evidence. Psychological Review, 347.

Sagone, E., \& Caroli, M. E. (2015). Positive personality as a predictor of high resilience in adolescence. Journal of Psychology and Behavioral Science, 45-53. 
Scheier, M. E., \& Carver, C. S. (1987). Dispositional optimism and physical well-being: The influence of generalized outcome expectancies on health. Journal of personality, 169-210.

Seligman, M. E. (1998). Building human strength: Psychology's forgotten mission. B DE BOOKS.

Seligman, M. E. (2017). La Autentica Felicidad. B DE BOOKS.

Vazquez, C., \& Hervas, G. (2009). Psicologia positiva aplicada. Bilbao: Desclee de Brouwer.

Vecina Jiménez, M. L. (2006). Emociones positivas. Pap. psicol, 9-17.

Vinaccia, S., Quinceno, J. M., \& San Pedro, E. M. (2007). Resiliencia en adolescentes. Revista colombiana de Psicologia, 139-146. 